Nova Biologica Reperta 7(3): 285-294 (2020)

Print ISSN: 2423-6330/Online ISSN: 2476-7115

https://nbr.khu.ac.ir; Kharazmi University Press; DOI: 10.29252/nbr.7.3.285

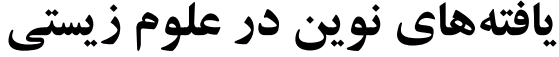

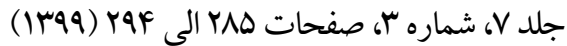

انتشارات دانشخاه خوارزمى

$$
\text { بررسى فون مارهاى منطقه شكارممنوع طالو و شيربند در استان سمنان، ايران }
$$

\author{
ويدا حجتى و مجتبى ديمه كار

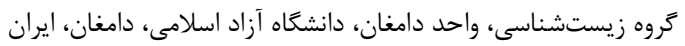

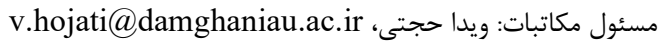

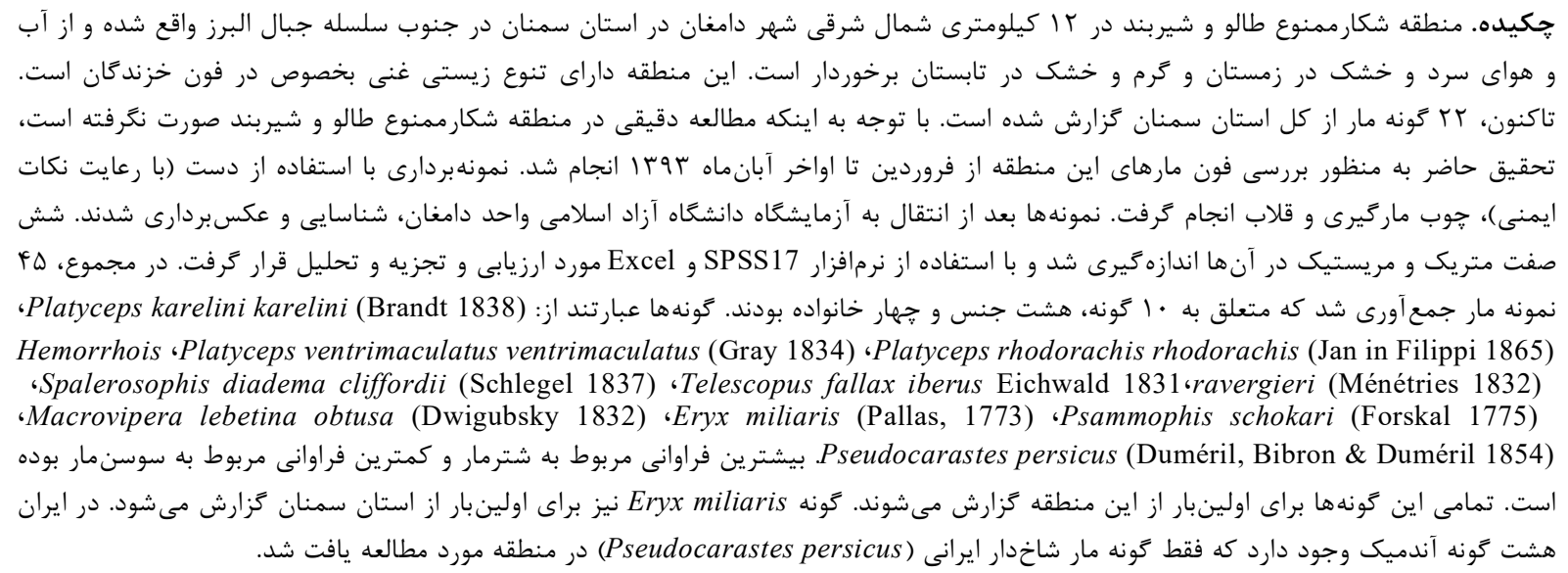

وازمهاى كليدى. تنوع زيستى، خزندكان، شكار، فون، مناطق حفاظتشده

\title{
The study of the snake fauna of Taloo and Shirband hunting prohibited area in Semnan Province, Iran
}

Vida Hojati \& Mojtaba Deymekar

Department of Biology, Damghan Branch, Islamic Azad University, Damghan, Iran

Correspondent author: Vida Hojati, v.hojati@damghaniau.ac.ir

\begin{abstract}
Taloo and Shirband hunting Prohibited area is located 12 kilometers North-East of Damghan in Semnan Province, south of the Alborz Mountain Chains. It has a cold and dry climate in the winter and warm and dry in the summer. This area has a rich biodiversity, especially in its reptilian fauna. 22 snake species were reported so far from Semnan Province. However, no comprehensice study was executed on the snake flora in Taloo and Shirband hunting prohibited area before the present study. Therfore, the present research was carried out in order to identify the snake fauna by collecting relevant specimens from March to late November of 2014. Sampling was done manually (with safety precautions) by sticking and hooking. The live specimens were identified and photographed and transferred to the laboratory of Islamic Azad University, Damghan Branch. Six metric and meristic traits were measured and analyzed using SPSS17 and Excel softwares. A total number of 45 snake specimens were collected which were belong to 10 species, 8 genera and 4 families, including: Platyceps karelini karelini (Brandt 1838), Platyceps rhodorachis rhodorachis, Platyceps ventrimaculatus ventrimaculatus (Spotted Flat-headed Snake); Hemorrhois ravergieri (Ravergier's Snake), Telescopus fallax iberus (Iberian Tiger-Snake), Spalerosophis diadema cliffordii (Clifford's Diadem Snake), Psammophis schokari (Schokari Sand Racer), Eryx miliaris (Dwarf Sand Boa), Macrovipera lebetina obtusa (Blunt Viper) and Pseudocarastes persicus (Persian False Horned Viper). The highest frequency belonged to Spalerosophis diadema cliffordii and the lowest frequency belonged to Telescopus fallax iberus. All species were reported for the first time from the studied area and Eryx miliaris was reported for the Semnan Province faunafor the first time. Out of 8 endemic species of snakes in Iran, only Pseudocarastes persicus was observed in the area.
\end{abstract}

Key words. biological diversity, fauna, hunting, protected areas, reptiles

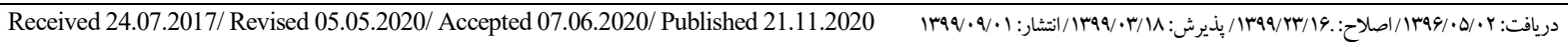


تابستانهاى معتدل است. از نظر وجود منابع آبى مىتوان كفت

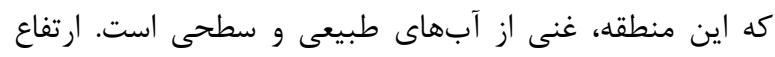

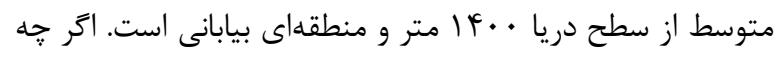
عمده يوشش گياهى از گياهان مرتعى است اما در ارتفاعات بالاتر

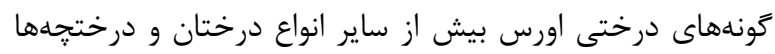

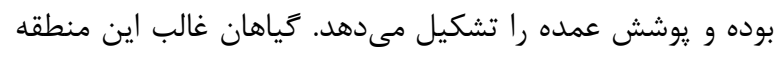

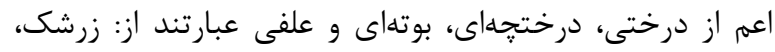

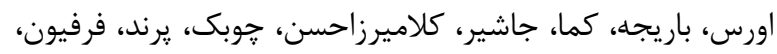

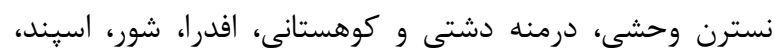

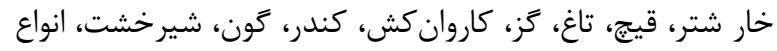

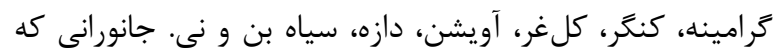

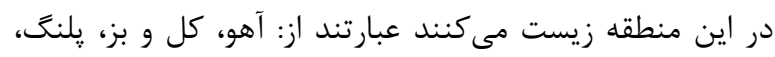

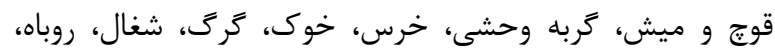

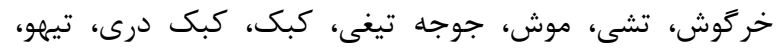

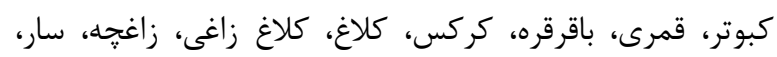

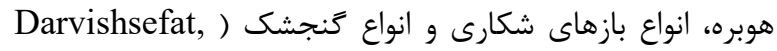

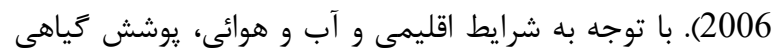

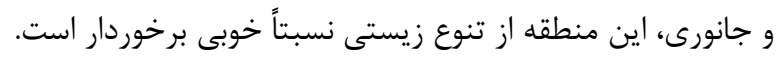

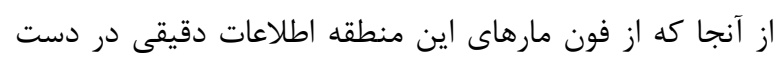

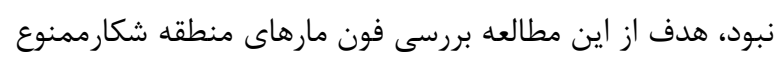
طالو و شيربند در استان سمنان است.

\section{مواد و روشها}

محدوده نمونهبردارى شامل منطقه شكارممنوع طالو و شيربند

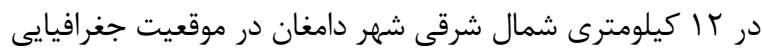

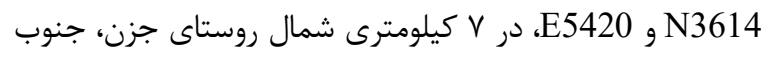
مسور كوه، شرق درياجه سد شهيد شاهجراغى است (شكل

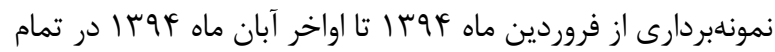

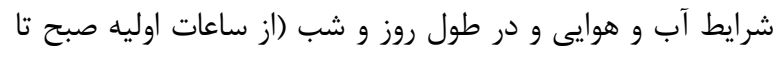

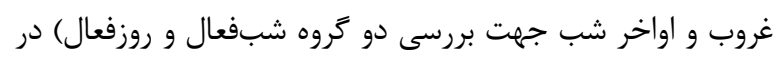

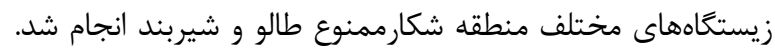

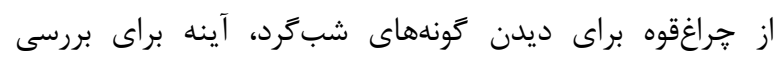

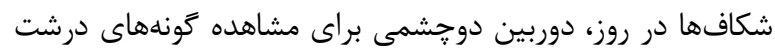

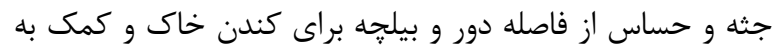

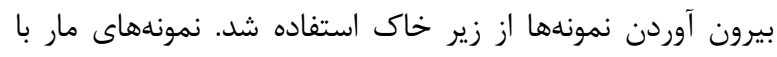
استفاده از دست (با رعايت نكات ايمنى و زيست زئن محيطى)،

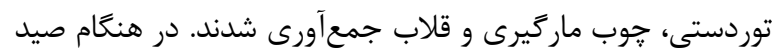

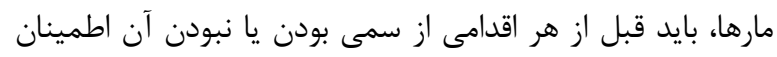

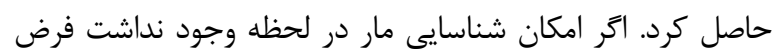

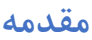

شرايط اقليمى و زيستكاههاى كوناكون در ايران موجب تنوع

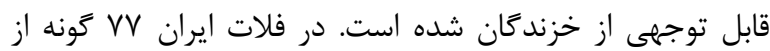

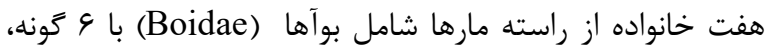
كلوبريده (Colubridae) با وج كونه، كبراها و مارهاى دريايى

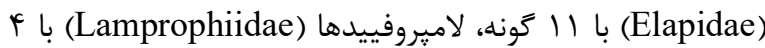
كونه، مارهاى خاكى (Leptotyphlopidae) با ب كونه، مارهاى كرمىشكل (Typhlopidae) با ب كونه و افعىها (Viperidae)

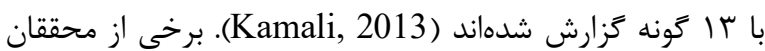

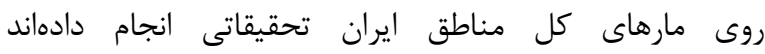
Farzanpey, 1990; Latifi, 2000; Kamali, 2013; ) منطعة). Safaei-Mahroo et al., 2015 منطقهاى روى فون مارهاى استان سمنان انجام شده و در حال

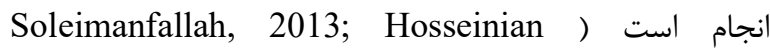
Yousefkhani et al., 2014; Derakhshanpour et al., .(2016; Zolfaghari et al., 2016 استان سمنان به دليل داشتن مناطق و موقعيت مناسب

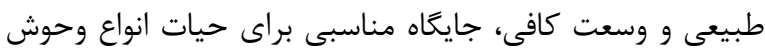

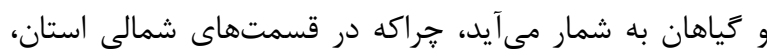

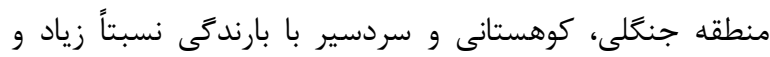
يوشش كياهى خوب، در قسمتهاى ميانى منطقه دشتى بارئا

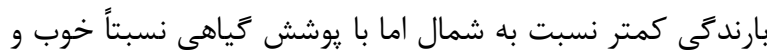

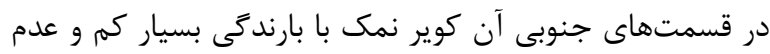

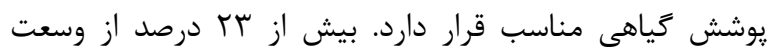

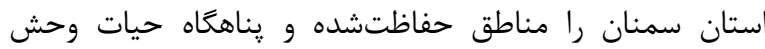
تشكيل مىدهد كه اين رقم در حدود هان درصد كل مناط مناطق ونان

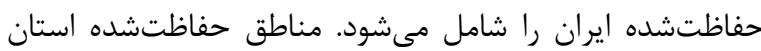

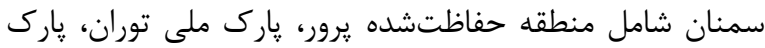

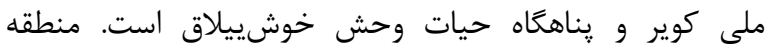

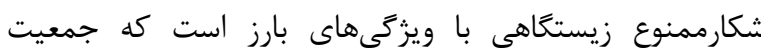

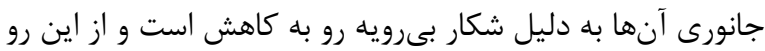

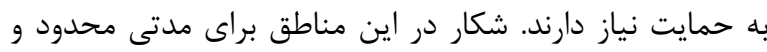

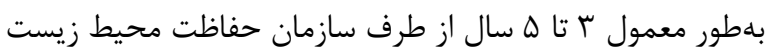

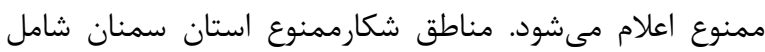

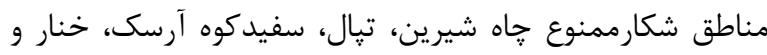

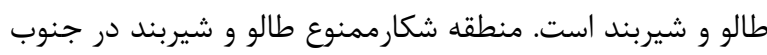
سلسله جبال البرز و شمال منطقه دشتى دامغان در استان

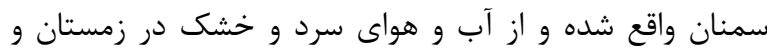

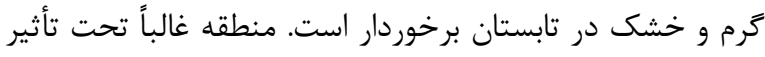

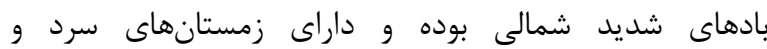


نمونهها به عنوان مدارك تحقيق در آزمايشگاه به طور زنده

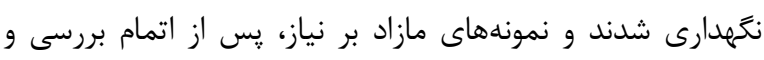
بيومترى رهاسازى شدند.

Latifi, ) براى شناسايى مارها از كليد شناسايى كتاب مارهاى ايران 2000) و اطلس رنكى خزند ائنان ايران (Mozaffari et al., 2017)

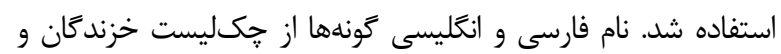
دوزيستان ايران (Safaei-Mahroo et al., 2015) اخذ شده است.

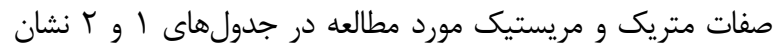

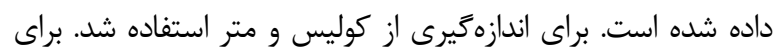
تجزيه و تحليل آمارى برروى صفات مورفومتريك و مريستيك از نرمافزار SPSS 17 و تجزيه
بر سمى بودن مار كذاشته شد و براى صيد آن ابتدا مار را از ناحيه سر مهار و سيس صيد انجام شد. يس از مشاهده هر يك از گونهها،

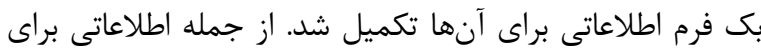
تك تك گونهها ثبت شد، نام گونه (كه با استفاده از يك كليد شناسايى معتبر شناسايى انجام شد)، نام محقق، تاريخ مشاهده

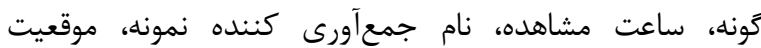
جغرافيايى (طول و عرض جغرافيايى)، شرايط جوى، دماى هوا، نوع

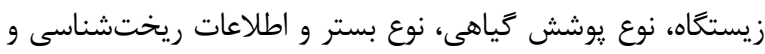

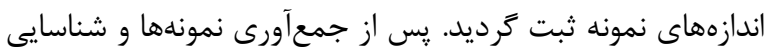
و ثبت آنها، تصاوير لازم از هر نمونه با دوربين عكاسى (دوربين و و لنز نرمال D Canon 90 •

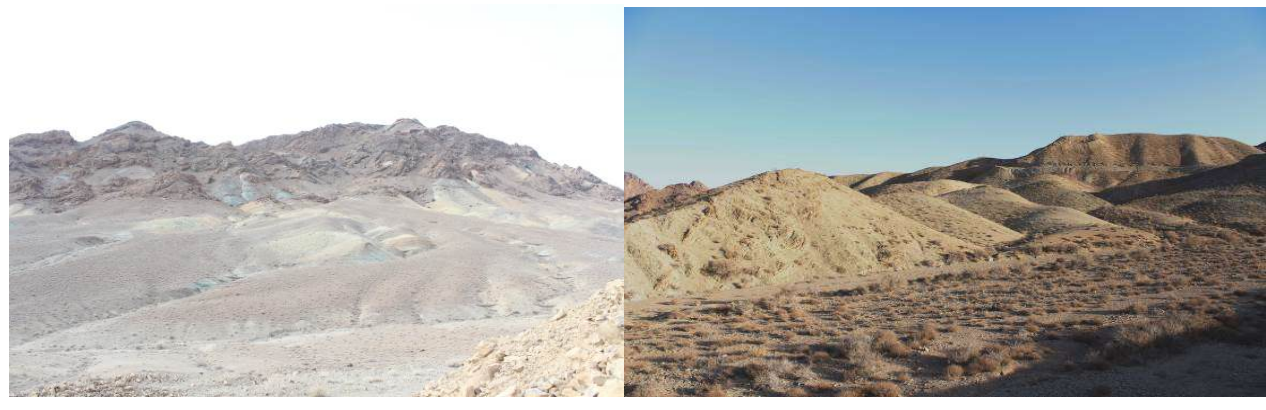

شكل ا- نماهايى از زيستگاههاى منطقه شكارممنوع طالو و شيربند

Fig. 1. Landscape photos of the Taloo and Shirnabd Prohibited Area habitats

جدول ا - صفات استاندارد در مارها

Table 1. Standard characters in the snakes studied

\begin{tabular}{|c|c|c|}
\hline توضيح & 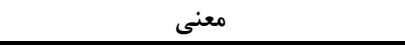 & علامت اختصارى \\
\hline از نوك يوزه تا اول مخرج & طول بدن (Snaout-Vent Length) & SVL \\
\hline از ابتداى مخرج تا انتهاى دم (دم غير ترميمى) & طول دم (Tail Length) & TL \\
\hline ت تعداد فلسهاى لب بالا & فلسهاى لب بالا (Supra-Labials) & SUL \\
\hline تعداد فلس هاى يشتى در يك رديف عرضى در قسمت ميانى بدن & تعداد فلسهاى يشتى (Dorsal Scales) & DS \\
\hline تعداد فلسهاى شكمى از اولين فلس شكمى تا مخرج & فلسهاى شكمى (Ventral Scales) & VS \\
\hline تعداد فلس هاى زيردمى از اولين فلس بعد از مخرج تا انتهاى دم & فلسهاى زيردمى(Subcaudal Scales) & SCS \\
\hline
\end{tabular}

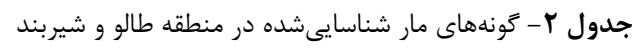

Table 2. Snake species Identified in Taloo and Shirband Prohibited Area

\begin{tabular}{|c|c|c|c|c|}
\hline خانواده & زيرخانواده & نام علمى كونه & 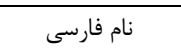 & تعداد نمونه \\
\hline \multirow{6}{*}{ Colubridae } & \multirow{6}{*}{ Colubrinae } & Telescopus fallax & سوسن مار & $r$ \\
\hline & & Platyceps rhodorachis & مار قيطانى & $\Delta$ \\
\hline & & Platyceps ventromaculatus & مار دستى & i \\
\hline & & Platyceps karelini karelini & مار خالدار & i \\
\hline & & Spalerosophis diadema & شترمار & $\Lambda$ \\
\hline & & Hemorrhois ravergieri & مار رِلنَّى & 4 \\
\hline Psammophidae & Psammophinae & Psammophis schokari & تيرمار & $r$ \\
\hline Boidae & Erycinae & Eryx miliaris & بواى شنى كوتوله & r \\
\hline \multirow{2}{*}{ Viperidae } & \multirow{2}{*}{ Viperinae } & Macrovipera lebetina obtusa & ( & $\checkmark$ \\
\hline & & Pseudocerastes persicus & مار شاخدار ايرانى & r \\
\hline
\end{tabular}


جدول r- آمار توصيفى در گونهاى مورد مطالعه.

Table 3. Descriptive statistics in the species studied.

\begin{tabular}{|c|c|c|c|c|}
\hline \multicolumn{5}{|c|}{ (سوسن مار) Telescopus fallax $(\mathrm{n}=2)$} \\
\hline انحراف معيار & خطاى معيار 土 ميانگين & حداكثر & حداقل & صفات \\
\hline r/AT & $\Delta r \pm r$ & $\Delta f$ & $\Delta \cdot$ & SVL \\
\hline $1 / .9$ & $\mid N / T \Delta \pm \cdot / V \Delta$ & 19 & $\mathrm{IV} / \mathrm{\Delta}$ & $\mathrm{TL}$ \\
\hline · & $\wedge \pm$ & $\Lambda$ & $\wedge$ & SUL \\
\hline . & $19 \pm$ & 19 & 19 & DS \\
\hline $\mid / 41$ & $r \mid \& \pm 1$ & riv & $r \mid Q$ & VS \\
\hline $\mid / 41$ & $\Delta V \pm 1$ & $\Delta \wedge$ & $\Delta \varphi$ & SCS \\
\hline \multicolumn{5}{|c|}{ ) Platyceps rhodorachis $(\mathrm{n}=5)$} \\
\hline $19 / 91$ & $V \Gamma / G \pm N / \Lambda$ & $9 \cdot 10$ & $\Delta \cdot$ & SVL \\
\hline$f / 99$ & $|V / r \cdot \pm r /| \cdot$ & $r M / Q$ & $11 / 0$ & TL \\
\hline$\cdot$ & $q \pm$ & 9 & 9 & SUL \\
\hline . & $19 \pm$ & 19 & 19 & DS \\
\hline $9|\Lambda|$ & $r r \mid \pm r / \cdot \Delta$ & rta & tif & VS \\
\hline $1 \cdot / r v$ & $\mid r \Delta / \Lambda \cdot \pm r / \varepsilon \psi$ & irs & $1 \cdot 1$ & SCS \\
\hline \multicolumn{5}{|c|}{ (مار دستى) Platyceps ventromaculatus $(\mathrm{n}=4)$} \\
\hline $11 / F F$ & $\varepsilon r / V \Delta \pm \Delta / V r$ & $\Lambda \cdot$ & $\Delta F$ & SVL \\
\hline$\Delta / \uparrow V$ & $r I \pm r / r r$ & rq & iv & $\mathrm{TL}$ \\
\hline$\cdot$ & $q \pm$ & 9 & 9 & SUL \\
\hline . & $19 \pm$ & 19 & 19 & DS \\
\hline$\cdot|\Lambda|$ & $r \cdots \pm \cdot / \kappa \cdot$ & $r \cdot 1$ & 199 & VS \\
\hline $\mathrm{V} / \mathrm{T} \mathrm{G}$ & $9 \Delta / r \Delta \pm r / 9 \Lambda$ & $1 \cdot r$ & $\Lambda$ & SCS \\
\hline \multicolumn{5}{|c|}{ (مار خال دار) Platyceps karelini karelini $(\mathrm{n}=4)$} \\
\hline$V Y / \cdot 1$ & $|q \psi \pm| q \mid \cdots$ & $r \cdot r$ & $\Delta \varphi$ & SVL \\
\hline rq/vi & $V \wedge / T \Delta \pm 19 / \wedge \Delta$ & $1 \cdot \Delta$ & $r$. & $\mathrm{TL}$ \\
\hline$\cdot$ & $q \pm$ & 9 & 9 & SUL \\
\hline . & $19 \pm$ & 19 & 19 & DS \\
\hline .190 & TYN/VD $\pm \cdot / F V$ & r. & rth & VS \\
\hline$\cdot / 90$ & $|r| / T \Delta \pm \cdot / F V$ & itr & $\pi$ & SCS \\
\hline \multicolumn{5}{|c|}{ (شترمار) Spalerosophis diadema $(\mathrm{n}=8)$} \\
\hline $1 r / \Delta r$ & $9 \Delta / 9 T \pm F / V \Lambda$ & AT & $F \Delta$ & SVL \\
\hline$r / r \cdot$ & $\mid Q / 9 \Lambda \pm 1 / 1 r$ & $r \cdot / \Delta$ & 11 & $\mathrm{TL}$ \\
\hline$\cdot / 91$ & $11 / 9 r \pm \cdot / 4 r$ & ir & 11 & SUL \\
\hline$\cdot \pi \omega$ & $r R / I r \pm \cdot / 1 r$ & $r \wedge$ & $r v$ & DS \\
\hline$\Lambda / \cdot \Lambda$ & $r$ rS \pm r/AS & rFD & rte & VS \\
\hline$T / V I$ & $\Lambda \Delta / \vee \Delta \pm \cdot / 9 \Delta$ & $9 \cdot$ & Ar & SCS \\
\hline \multicolumn{5}{|c|}{ (مار پلنكى) Hemorrhois ravergieri $(\mathrm{n}=6)$} \\
\hline$V / 9 F$ & 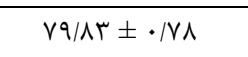 & $9 \cdot 10$ & $V \cdot / \Delta$ & SVL \\
\hline$\cdot$ & $Y F / Q \pm|/ r|$ & $r q / \Delta$ & rt & $\mathrm{TL}$ \\
\hline · & $9 \pm$ & 9 & 9 & SUL \\
\hline$\cdot$ & 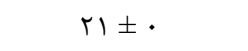 & r) & rI & DS \\
\hline$r \varphi / V V$ & $\mid \wedge 9 / \Delta \pm 1 \Delta / \cdot 1$ & rl. & 110 & VS \\
\hline$\Delta / f\rangle$ & $\wedge \varepsilon /|\vee \pm r / r|$ & ar & v१ & SCS \\
\hline
\end{tabular}




$$
\text { جدول r- ادامه. }
$$

Table 3. continue.

(تيرمار) Psammophis schokari $(\mathrm{n}=3)$

\begin{tabular}{|c|c|c|c|c|}
\hline$\Lambda / \Lambda \Lambda$ & $V \Gamma / Q \pm \Delta / I T$ & $\vee 9 / \Delta$ & GT/D & SVL \\
\hline f & $19 \pm r / r$. & r & 10 & $\mathrm{TL}$ \\
\hline · & $9 \pm$. & 9 & 9 & SUL \\
\hline . & IV \pm & iv & iv & DS \\
\hline$N / \cdot r$ & $\mid \Lambda r / r r \pm r / q r$ & 19. & IVF & VS \\
\hline $19 / 9 V$ & $q r \pm 11 / \Delta r$ & 110 & v9 & SCS \\
\hline \multicolumn{5}{|c|}{ Eryx miliaris $(\mathrm{n}=3)$} \\
\hline $1 \cdot 1 \cdot 1$ & L $\pm \Delta / V \Lambda$ & $\Delta 1$ & rt & SVL \\
\hline$\cdot 10$ & $r / \Delta \pm \cdot / r \Lambda$ & r & r & $\mathrm{TL}$ \\
\hline 1 & $q q \pm \cdot / \Delta V$ & $\Delta \cdot$ & $\psi_{\wedge}$ & DS \\
\hline$r / \cdot r$ & $190 / 9 V \pm r / r r$ & $r \cdot$. & 195 & VS \\
\hline$r|\Delta|$ & $r \& / 9 V \pm 1 / 4 \Delta$ & rq & rF & SCS \\
\hline \multicolumn{5}{|c|}{ (كرزه مار) Macrovipera lebetina obtusa $(n=7)$} \\
\hline$r V / V \cdot$ & $V \wedge / Y I \pm 1 \cdot / 49$ & $1 \pi$ & FV & SVL \\
\hline T/DT & $9 / \Delta V \pm \cdot / 9 \Delta$ & if & $4 / 0$ & $\mathrm{TL}$ \\
\hline$\cdot / r V$ & $1 \cdot / 1 F \pm \cdot / 1 F$ & 11 & $1 \cdot$ & SUL \\
\hline$\cdot / 9 \mathrm{~V}$ & $r F / F T \pm \cdot / K \varepsilon$ & $r \Delta$ & r & DS \\
\hline $9 / \mu Y$ & $|99 / r| \pm r / \Delta r$ & $I \vee \wedge$ & 149 & VS \\
\hline$\Delta / 94$ & $r T / r q \pm r / / r$ & $\Delta \cdot$ & ra & SCS \\
\hline \multicolumn{5}{|c|}{ (مار شاخدار ايرانى) Pseudocerastes persicus $(\mathrm{n}=3)$} \\
\hline$T|/ D|$ & $\Delta I / A r \pm I r / K r$ & 90 & rV & SVL \\
\hline$r / 4 \&$ & $\Delta / A r \pm 1 / \mathcal{K} T$ & $V / \Delta$ & r & $\mathrm{TL}$ \\
\hline $1 / 10$ & $11 / 9 V \pm \cdot / 99$ & ir & 11 & SUL \\
\hline$\cdot \mid \Delta V$ & מr/ ש & ry & r & DS \\
\hline $\mathrm{V} / \mathrm{gr}^{2}$ & $|\Delta| / \varepsilon V \pm f / F \mid$ & 19. & 140 & VS \\
\hline$r$ & $R r \pm 1 / r r$ & $\forall \Delta$ & rq & SCS \\
\hline
\end{tabular}

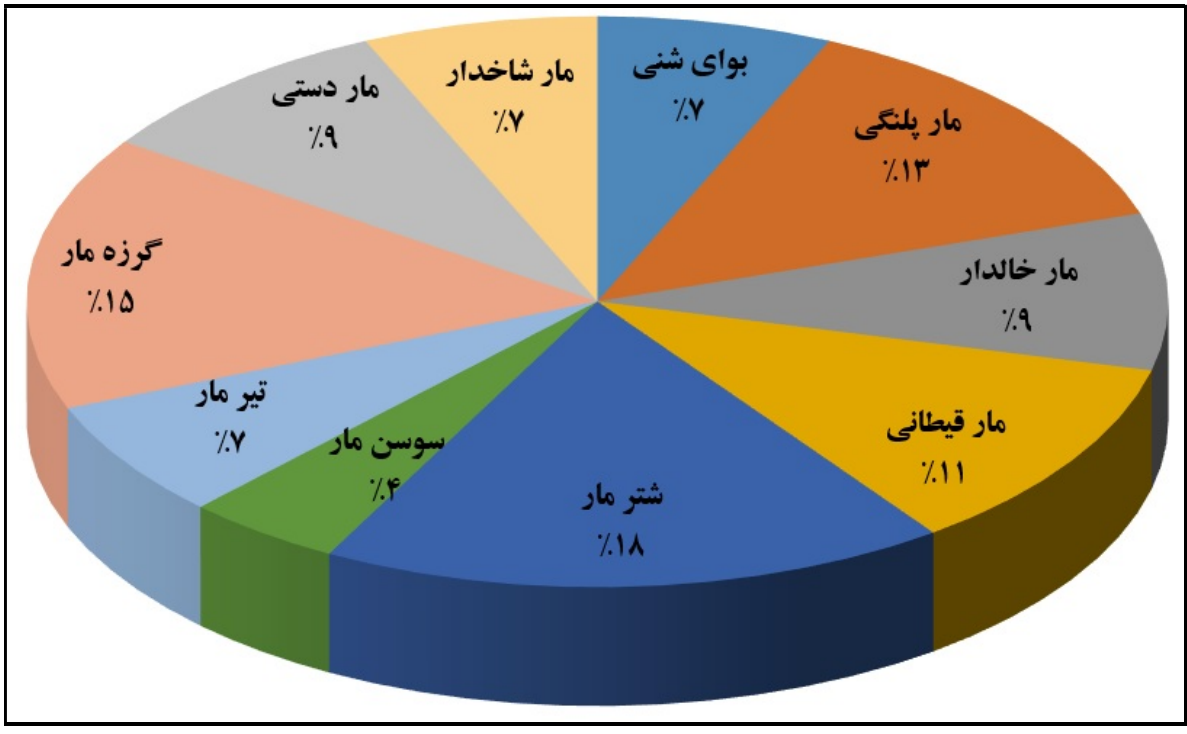

شكل r - درصد فراوانى مارهاى مشاهده شده در منطقه شكارممنوع طالو و شير بند.

Fig. 2. Frequency percentages of snakes observed in Taloo and Shirband Hunting Prohibited Area. 


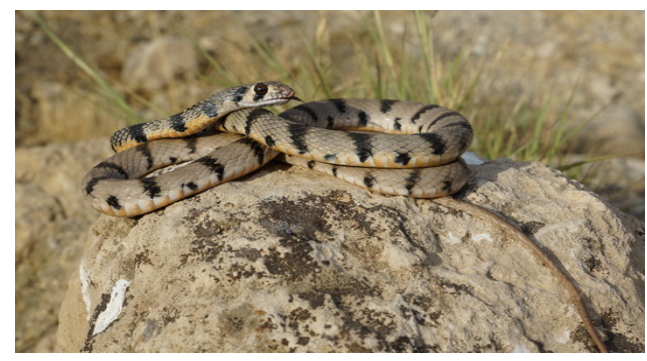

شكل ץ- مار خال دار.

Fig. 4. Platyceps karelini karelini.

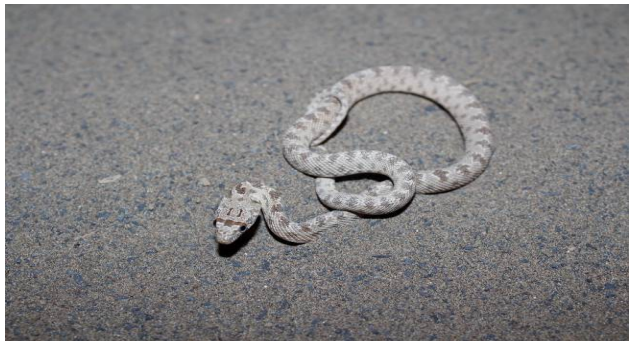

شكل 9- شترمار.

Fig. 6. Spalerosophis diadema cliffordii.

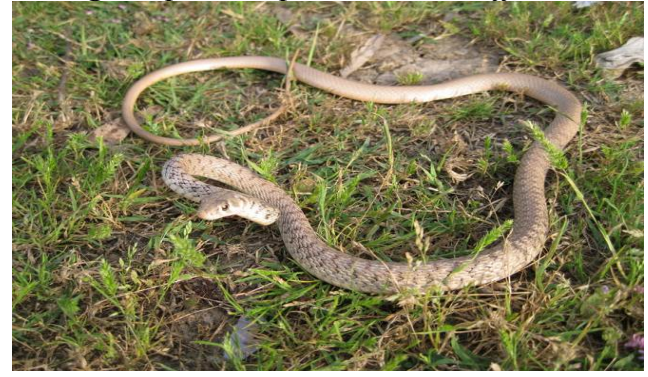

شكل 1 - مار دستى.

Fig. 8. Platyceps ventrimaculatus.

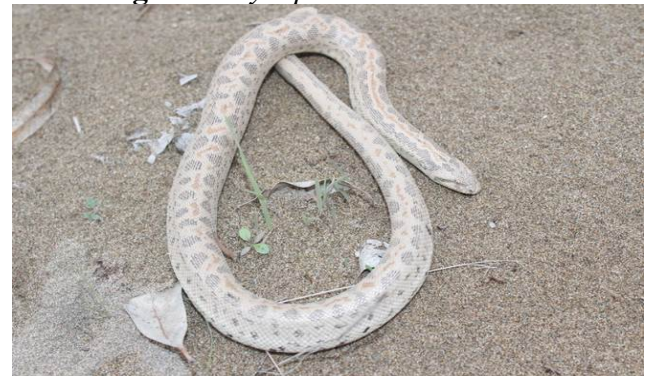

شكل •ا- بواى شنى كوتوله.

Fig. 10. Eryx miliaris.

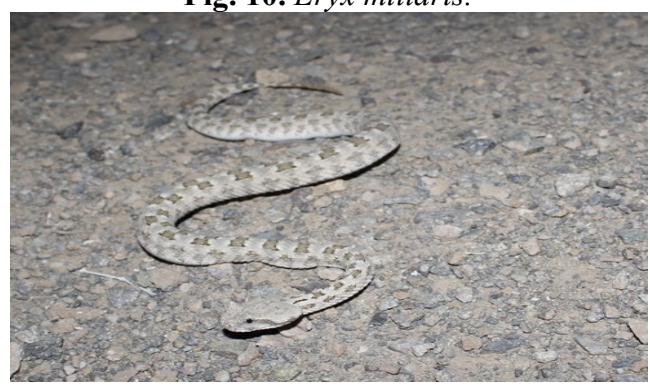

$$
\text { شكل rا - مار شاخدار. }
$$

Fig. 12. Pseudocerastes persicus.

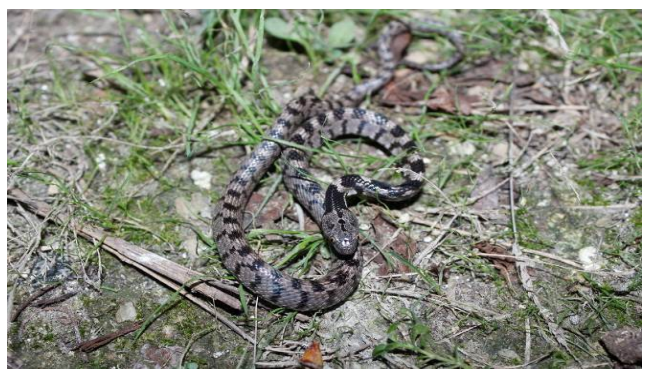

$$
\text { شكل r - سوسن مار. }
$$

Fig. 3. Telescopus fallax iberus.

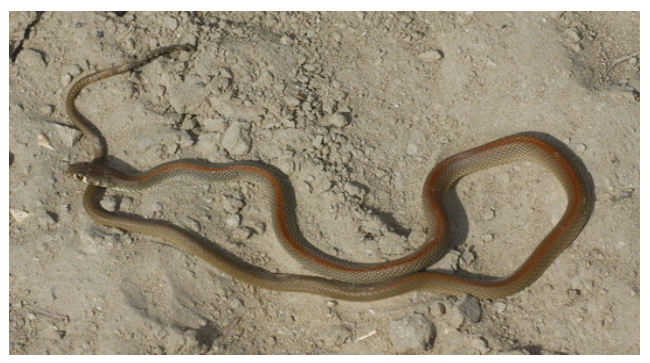

$$
\text { شكل ه- مار قيطانى. }
$$

Fig. 5. Platyceps rhodorachis rhodorachis.

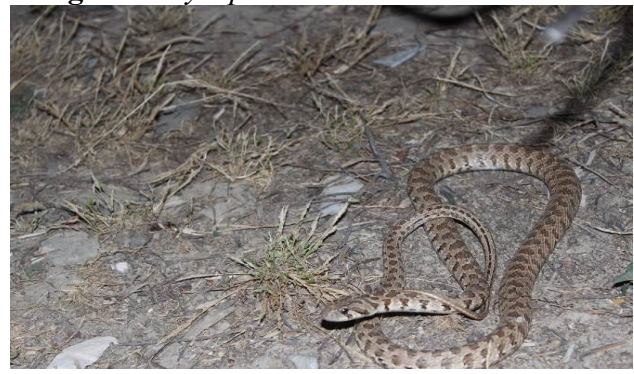

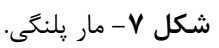

Fig. 7. Hemorrhois ravergieri.

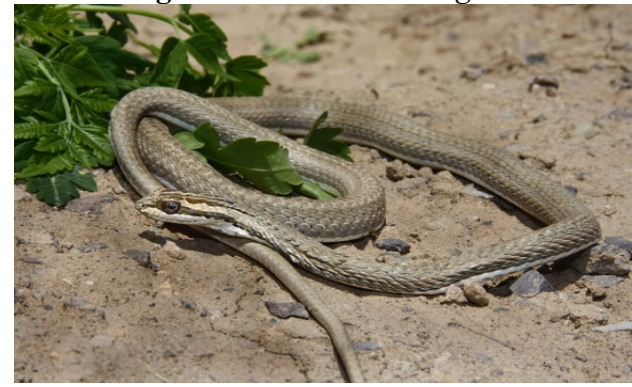

$$
\text { شكل 9- تيرمار. }
$$

Fig. 9. Psammophis schokari.

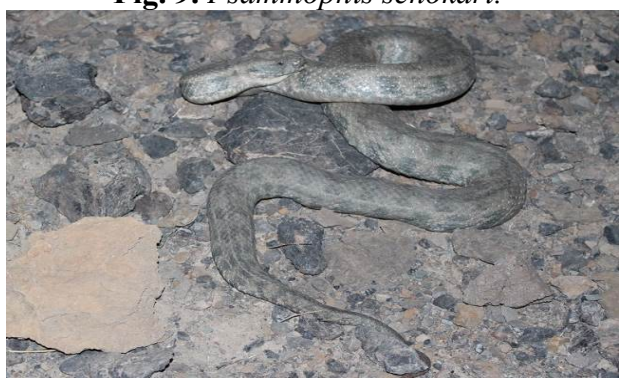

$$
\text { شكل ال1 - كرزه مار. }
$$

Fig. 11. Macrovipera lebetina obtuse. 


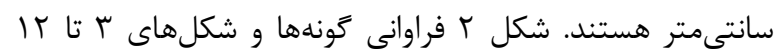

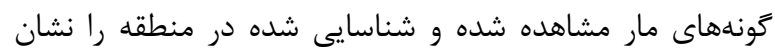

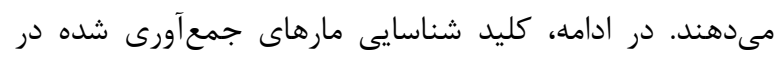
منطقه شكارممنوع طالو و شيربند نيز ارائه شده است دارئ

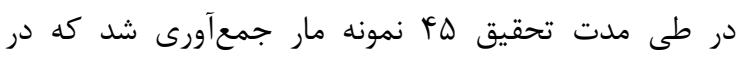

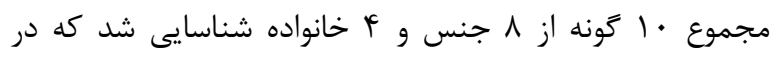

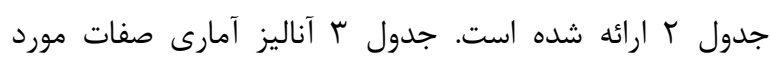

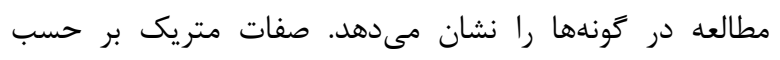
كليد شناسايى مارهاى منطقه شكارممنوع طالو وشير بند سمنان

$(Y)$ la

( $(\mathrm{r})$ l - Ib

Spalerosophis diadema cliffordii ra (ए)

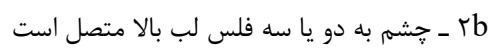

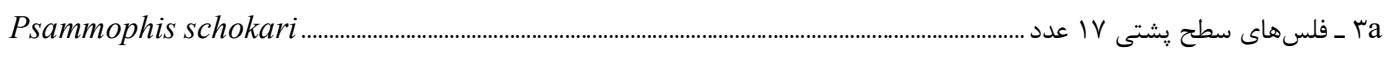

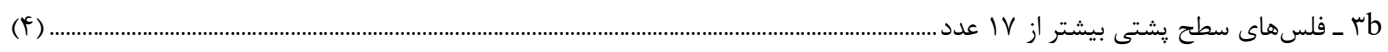

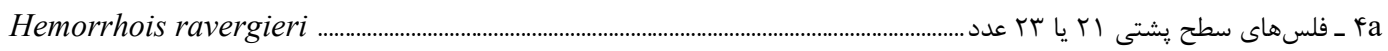

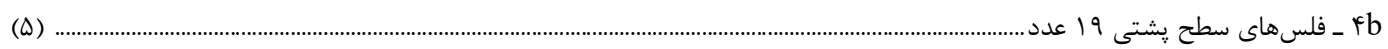

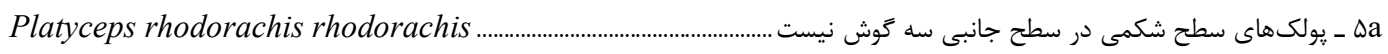

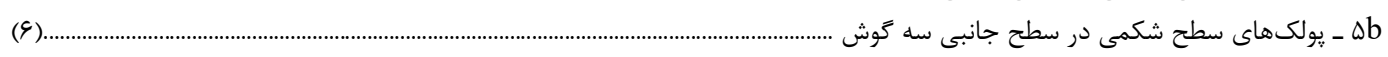

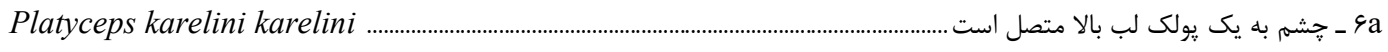

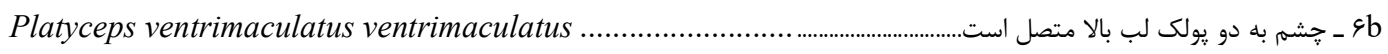

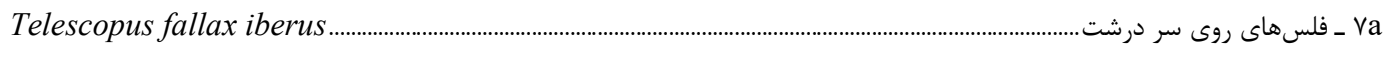

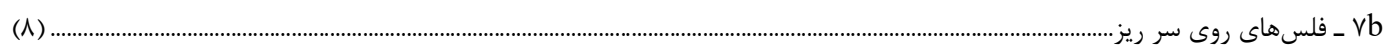

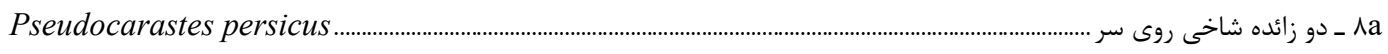

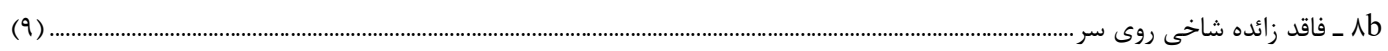

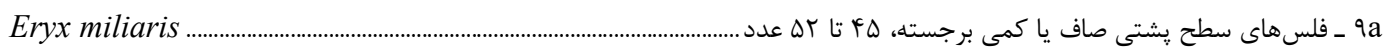

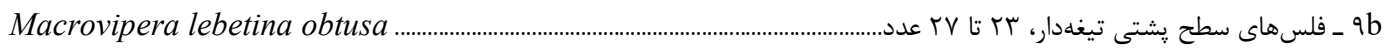

\section{Elaphe „Elaphe dione “Coluber ventrimaculatus} Spalerosophis “Natrix natrix ،quatuorineata sauromates Spalerosophis diadema schirazianus diadema clifordi Psammophis schokari Psammophis lineolatus Naja ،Telescopus rhinopoma ،Telescopus fallax iberus Vipera lebetina ‘Echis carinatus sochureki ،naja oxiana Agkistrodon, Pseudocerastes persicus persicus ،obtusa intermedicus caucasicus

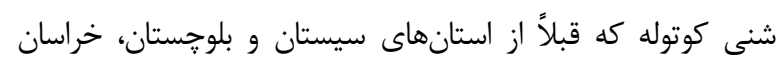
جنوبى، رضوى و شمالى، كلستان، مازندران، مركزى، همدان، اصفهان،

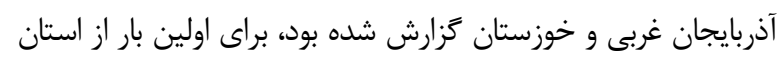

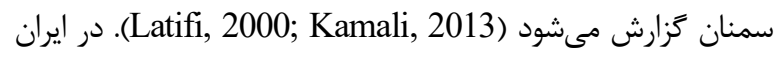

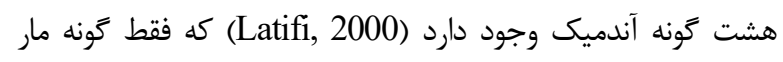
شاخدار ايرانى (Pseudocarastes persicus) در منطقه مورد مطالعه يافت شد. ياخ خانواده كلوبريده بزركترين خانواده مارها از لحاظ تعداد كَنه در

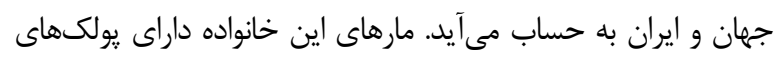

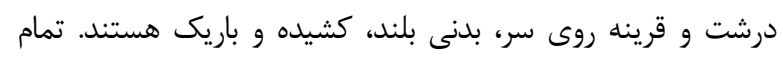

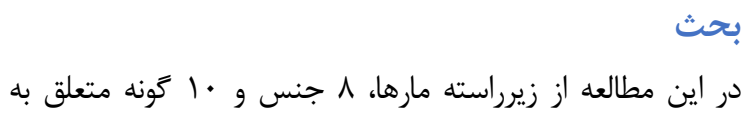
جهار خانواده Psammophidae Colubridae، بiperidae

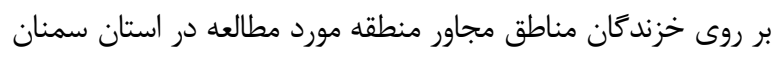

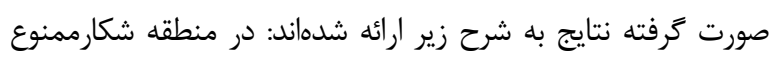

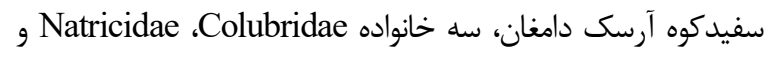
Viperidae (Soleimanfallah, 2013) يرور، سه خانواده Niperidae , Natricidae Colubridae كزارش شدند. در تحقيقاتى كه

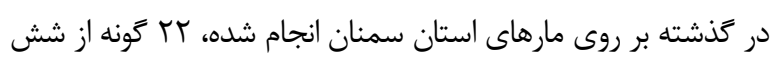
خانواده Colubridae، Latifi, 2000; ) كزارش شده است Viperidae Natricidae شامل شامل Eryx jaculus familiaris ، Eryx jaculus jaculus ‘Colube karelini ،Coluber schmidti ،Coluber jugularis ،Coluber rhodorachis ‘Coluber ravergieri ravergieri 
عدد (Latifi, 2000)؛ تعداد فلس لب بالا 9-1 عدد، فلسهاى يشتى

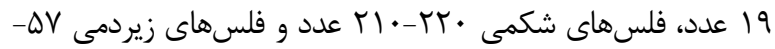
צ عدد (Soleimanfallah, 2013)؛ تعداد فلس لب بالا 1 عدد، فلسهاى يشتى 19 عدد، فلسهاى شكمى

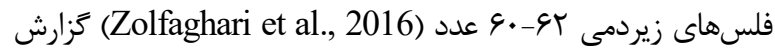
شده است.

Platyceps karelini ) بزرَترين نمونه بررسىشده از مار خال

(karelini

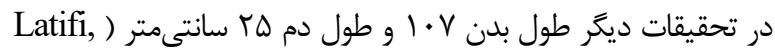
2000)؛ طول بدن فه و طول دم 9 (اسانتىمتر ( Derakhshanpour

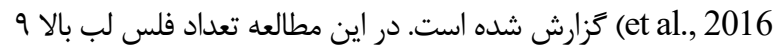

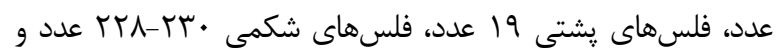

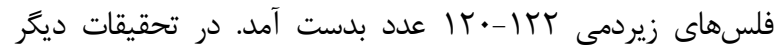
تعداد فلس لب بالا 9 عدد، فلسهاى يشتى 19 عدد، فلسهاى

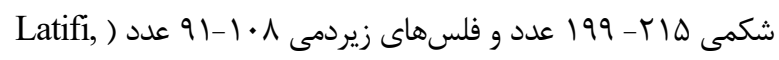
2000)؛ تعداد فلس لب بالا 1 عدد، فلسهاى يشتى 19 عدد،

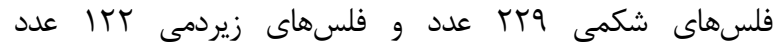
(Derakhshanpour et al., 2016)

در اين مطالعه، بزرگترين نمونه مار گِلنكى ( Hemorrhois (ravergieri بوده اما در تحقيقات ديخر طول بدن 109 و طول دم TV سانتىمتر

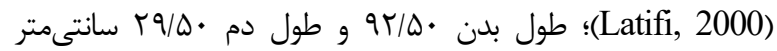
(Derakhshanpour et al., 2016) • • ه/ اين مطالعه تعداد فلس لب بالا 9 عدد، فلسهاى يشتى آ إ عدد،

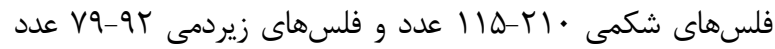
شمارش شده است. در تحقيقات ديخر تعداد فلس لب بالا • 1 -9 عدد،

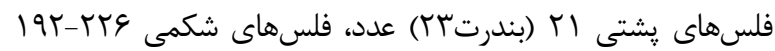

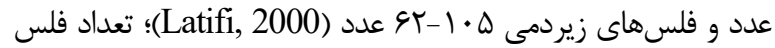

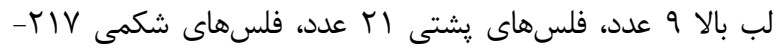

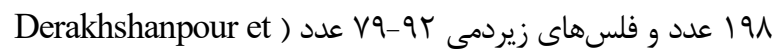

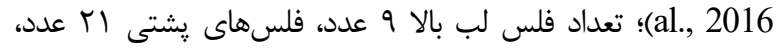

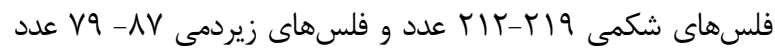
(Zزارش شده است. (Zolfaghari et al., 2016)

Platyceps ) بزرگترين نمونه بررسىشده از مار قيطانى (rhodrachis rhodorachis

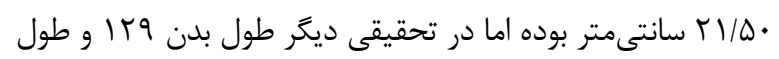
دم ها سانتىمتر (Latifi, 2000) گزارش شده است. در اين مطالعه تعداد فلس لب بالا 9 عدد، فلسهاى يشتى 19 عدد،

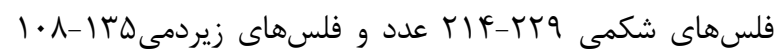

مارهاى اين خانواده به استثناى جهار گ گونه آلوسر، افعىيلنگى، سوسن مار، و افعى سوسن همكَى داراى مردمك گَرد هستند. در اين مطالعه از خانواده كلوبريده، شش تونه ،Platyceps rhodorachis rhodorachis ،karelini Platyceps ventrimaculatus ،Hemorrhois ravergieri Telescopus fallax iberus ،ventrimaculatus Spalerosophis diadema cliffordii و شناسايى شد. از منطقه شكارممنوع سفيد كوه آرسك، جهار زونه Telescopus

و Eirenis collaris ،Platyceps najadum fallax iberus Platyceps ventrimaculatus (Soleimanfallah, 2013). از منطقه حفاظتشده يرور، شش گونه Platyceps ،Dolichophis schmidti ،Platyceps karelini Telescopus fallax ،ventrimaculatus ventrimaculatus Elaphe dione و Platyceps najadum ،iberus است (Derakhshanpour et al., 2016). بر اساس مطالعات گذشته بر روى مارهاى ايران در مجموع كونه

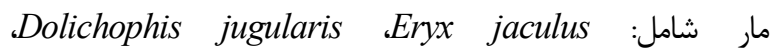
Hemorrhois .Platyceps karelini Dolichophis schmidti Platyceps Platyceps rhodorachis ravergieri Elaphe ‘Elaphe dione ،Natrix natrix ،vetrimaculatus Psammophis Spalerosophis diadema sauromates Telescopus ‘Psammophis schokari ،lineatus Echis, Naja oxiana ،Telscopus rhinopoma fallax Latifi, ) از استان سمنان گزارش شده است carinatus sochureki در مطالعه ديكرى، گونه Boiga trigonata نيز از اين استان

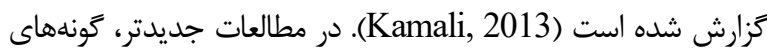
و Platyceps najadum ،Eirenis punctatolineatus نيز از استان سمنان گزارش شده است Lytrhynchus ridgewayi .(Safaei Mahroo et al., 2015) بزرگترين نمونه سوسنمار (Telescopus fallax iberus) داراى طول بدن QF و طول دم 19 سانتىمتر بوده ولى در مطالعات ديخر

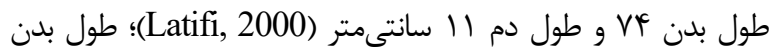

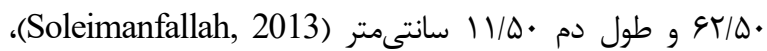

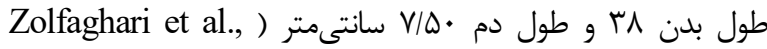
2016) گزارش شده است. در اين مطالعه تعداد فلس لب بالا ^ عدد، فلسهاى يشتى 19 عدد، فلسهاى شكمى

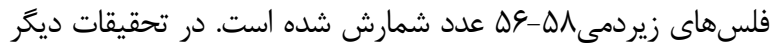

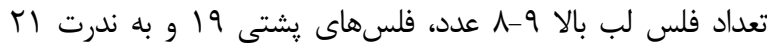

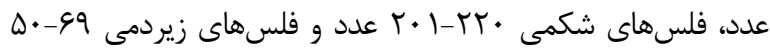




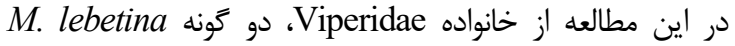
شناسايى شد. در تحقيق ديخرى از منطقه

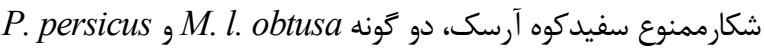
(Soleimanfallah, 2013)؛ در منطقه حفاظتشده يرور، دو كونه (Derakhshanpour et al., 2016) G. halys و M. lebetina كزارش شد؛ اما در تحقيقاتى كه بر روى مارهاى استان سمنان انجام شده جهار كونه P. persicus M. lebetina E. carinatus (Kamali, 2013; Latifi, 2000) G. halys

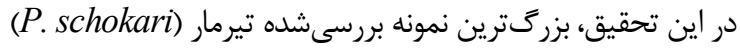
داراى طول بدن •V9/D و طول دم بآ سانتىمتر بوده كه در مطالعات

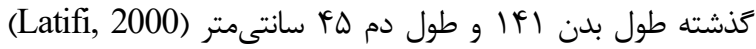

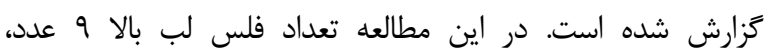

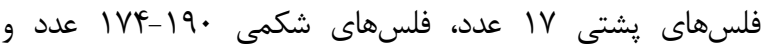
فلسهاى زيردمى || || V9 عدد كزارش شده است. همجنين تعداد

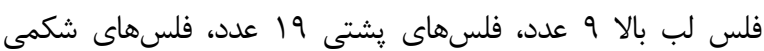

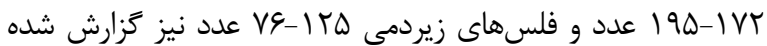

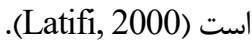

در اين تحقيق، بزركترين نمونه بواى شنى (E. j. familiaris) داراى طول بدن اله و طول دم ז سانتىمتر بوده كه قبلاً طول بدن

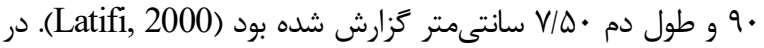

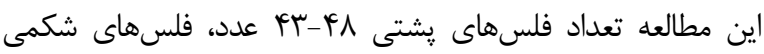
عدد و فلسهاى زيردمى q 9-TV-IVD

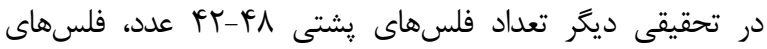

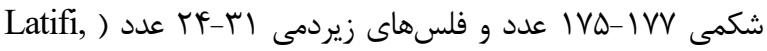
2000) كزارش شده است.

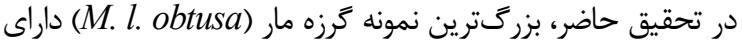

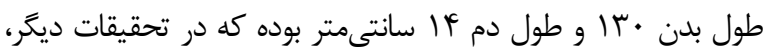
طول بدن 191 و طول دم • r سانتىمتر (Latifi, 2000)؛ طول بدن

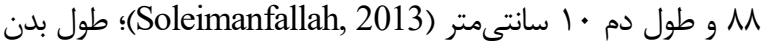

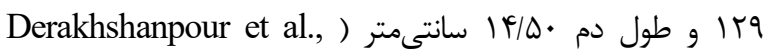
2016) كزارش شده است. در اين مطالعه، تعداد فلس لب بالا ||- |-

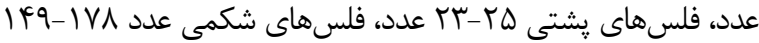

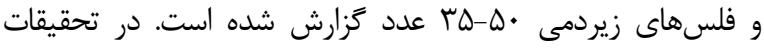

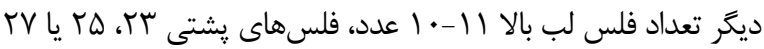

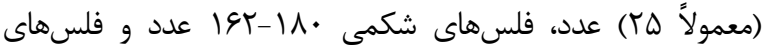

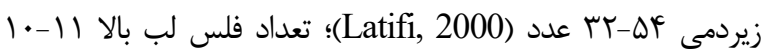

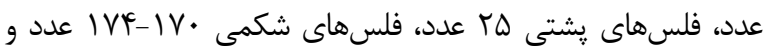

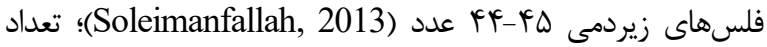

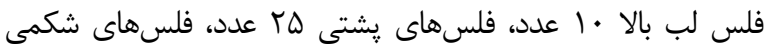

عدد شمارش شده است. در تحقيقات ديخر تعداد فلس لب بالا

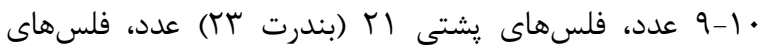

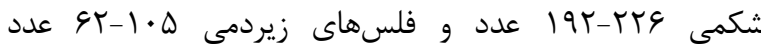
(Latifi, 2000)؛ گزارش شده است.

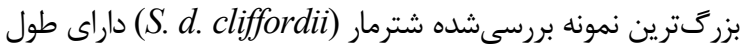

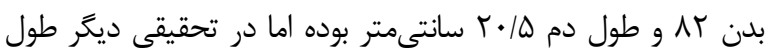

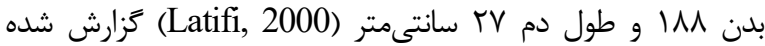

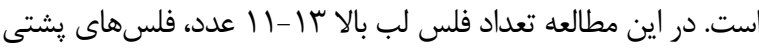

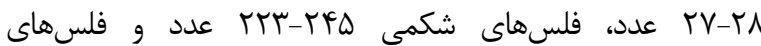

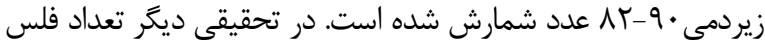

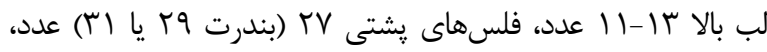
فلسهاى شكمى YY (Latifi, 2000)

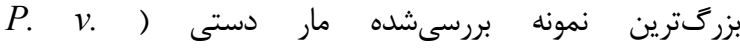
(ventrimaculatus

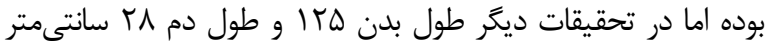

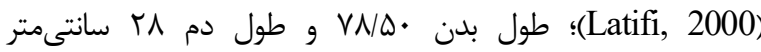
(Derakhshanpour et al., 2016)

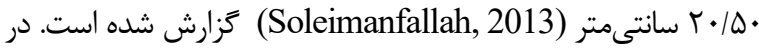
اين مطالعه تعداد فلس لب بالا 9 عدد، فلسهاى يشتى 19 عداد، 19 عداد

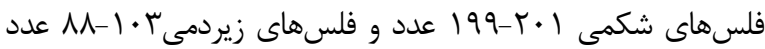
شمارش شده است. در تحقيقات ديكر تعداد فلس لب بالا 9 عدد،

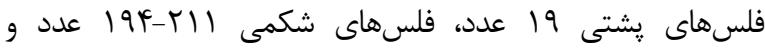

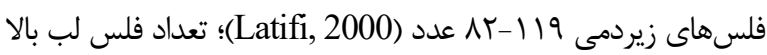

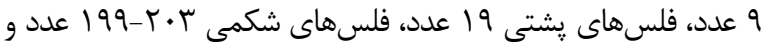

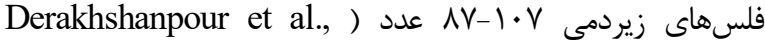
2016)؛ تعداد فلس لب بالا 9 عدد، فلسهاى يشتى 19 عدد، فلسهاى شكمى TII عدد و فلسهاى زيردمى بأ عدد (Soleimanfallah, 2013)

در اين مطالعه از خانواده Psammophidae يك گَّونه Psammophis schokari (Latifi, 2000; Kamali, 2013)، علاوه بر اين كَونه، كونه Psammophis lineolatus Safaei-Mahroo et al., 2015 Malpolon insignitus خانواده Erycidae، كونه E. miliaris شناسايى شد. در تحقيقات

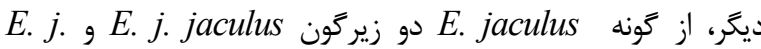
E. j. turcicus (Latifi, 2000) familiaris كزارش شده (Kamali, 2013; Safaei-Mahroo et al., 2015) 
برناملريزىهاى كوتاه و بلندمدت به نحوى از روند رشد منفى كاهش

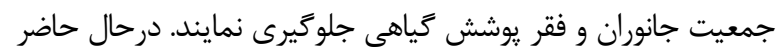

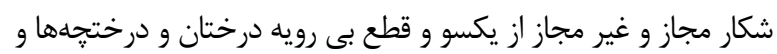

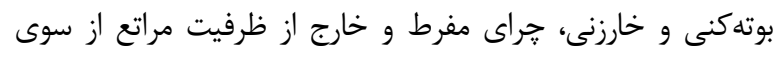

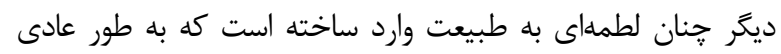

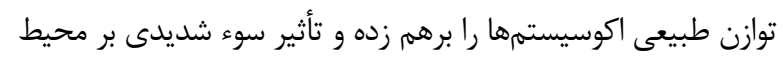

زيست موجوداتى وارد مىسازد كه وابستخى اكولوزى كاملى به ديگر

عوامل حيات دارند. اميد است با بررسىهاى همه جانى جانبه منطقه مذكور

نيز با ارتقاء به سطوح بالاتر از جمله منطقه حفاظتشده جزء مناط اطنق

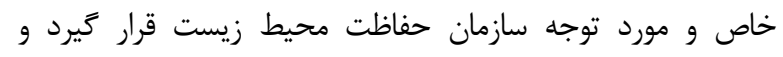

يايغاهى جهت انجام امور تحقيقاتى و مطالعاتى گردد.

$$
\text { سباسگزارى }
$$

نويسندكان مقاله از آقاى رضا بابايى سواسرى براى كمكهاى

بى دريغ در جمعآورى نمونهها و آقاى فريبرز حيدرى در تهيه تصاوير،

مسئولين سازمان حفاظت محيط زيست شهرستان دامغان و همجرين

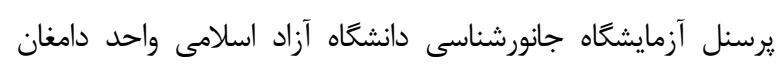

$$
\text { قدردانى مىنمايند. }
$$

\section{REFERENCES}

Darvishsefat A. 2006, Atlas of Protected Areas of Iran. University of Tehran Publication, Tehran. pp: 170.

Derakhshanpour, Z., Hojati, V. \& Abbaspour, H. 2016. The faunestics study of snakes of Parvar Prohibited Area in Semnan Province. Journal of Animal Environment 8: 249-258.

Farzanpey, R. 1990. Ophiology. Tehran: Central University. Publications no. 523, Biology 13: 1-12. (in Persian).

Hosseinian Yousefkhani, S.S., Yousefi. M, Khani, A. \& Rastegar Pouyani, E. 2014. Snake fauna of Shirahmad wildlife refuge and Parvand protected area, Khorasan Razavi province, Iran. Herpetological Notes 7: 75-82.

Kamali, K. 2013. A Field Guide for the reptiles and amphibians of Iran. Iranshenasi Publication, $1^{\text {st }}$ edition. pp: 368 .

Latifi, M., 2000. The snakes of Iran. Iran department of the environment, $3^{\text {rd }}$ edition, Tehran. pp: 478.

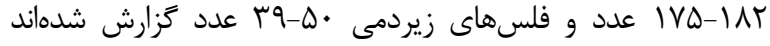

(Derakhshanpour et al., 2016) بزرگترين نمونه بررسىشده از مار شاخدار (P. persicus) داراى طول بدن له و طول دم • V/D سانتىمتر بوده اما در تحقيقات ديگر

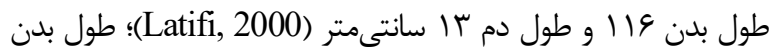

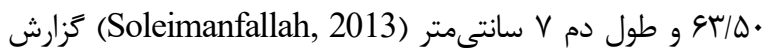

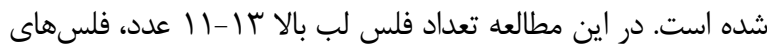

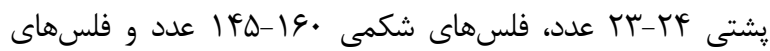

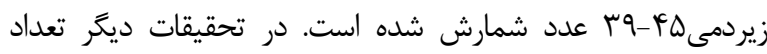

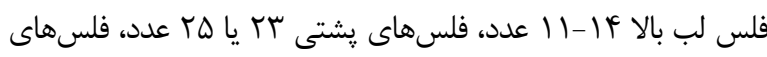

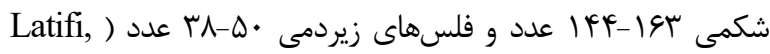

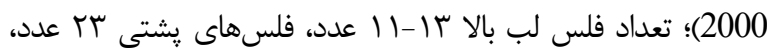
فلسهاى شكمى وها (Soleimanfallah, 2013)

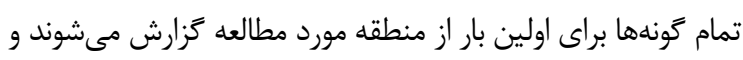
تنها يك گونه بومى ايران در منطقه مشاهده شد.

تأثير سوء فعاليتهاى انسانى و بهرهبردارى ناصحيح و غير اصولى از

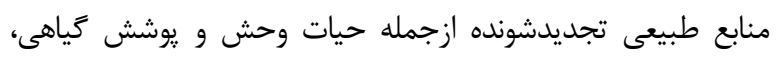

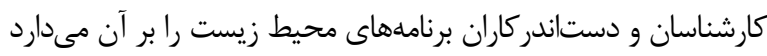

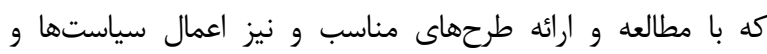

Mozaffari, O., Kamali, K. \& Fahimi H. 2017. Atlas of reptiles of Iran. Jahad Daneshgahi-Alborz Province (Kharazmi) Publication, $1^{\text {st }}$ edition. pp: 360.

Safaei-Mahroo, B., Ghaffari, H., Fahimi, H., Broomand, S., Yazdanian, M., Najafi Majd, E., Hosseinian Yousefkhani, S.S., Rezazadeh, E., Hosseinzadeh, M.S., Nasrabadi, R., Rajabizadeh, M., Mashayekhi, M., Motesharei, A., Naderi, A. \& Kazemi, S.M. 2015. The herpetofauna of Iran: checklist of taxonomy, distribution and conservation status. Asian Herpetological Research 6: 257-290.

Soleimanfallah, D. 2013. The Study of amphibians and reptiles fauna in Sefid Kouh-Aresk No-Hunting Area in Semnan Province. Sc. Thesis, Islamic Azad University, Damghan Branch. pp: 117.

Zolfaghari, N., Hojati, V. \& Shajiee, H. 2016. The faunestics study of the amphinians and snakes of Hezar Jarib No Hunting Area in Mazandaran Province. Journal of Animal Environment 8: 251258.

How to cite this article:

Hojati, V. \& Deymekar, M. 2020. The study of the snakes fauna of Taloo and Shirband hunting prohibited area in Semnan province. Nova Bioloica Reperta 7: 285-294. (In Persian).

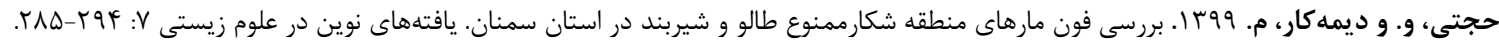

